\title{
The Effect of Individual Stone Dimensions on Stone Passage Rates
}

\author{
(D) Dwayne Chang1,2, (1) Mikhail Lozinskiy ${ }_{1}^{1}{ }^{2}$, (D) Angela Jacques3 , (1) Melvyn Kuan1,2 \\ 'Department of Urology, Rockingham General Hospital, Rockingham, Western Australia \\ 2 Urology Department, Fiona Stanley Hospital, Murdoch, Western Australia \\ 3Institute for Health Research, University of Notre Dame Australia, Fremantle, Western Australia
}

\section{What's known on the subject? and What does the study add?}

It is well known that the size of a ureteric stone affects the required for spontaneous stone passage. However, it is uncertain which of the three dimensions of a ureteric stone best correlates with the time required for spontaneous stone passage. Our pilot study investigated this clinical question. Although elongated stones required marginally less time to pass spontaneously, our results did not demonstrate a statistically significant correlation between the time required for ureteric stones to pass spontaneously with any particular stone dimension.

\section{Abstract}

Objective: The aim of this study is to determine the relationship between each of the three dimensions of a ureteric stone and the passage rate and to identify ureteric stones with an oval/elongated shape and determine whether the stone passage rate was most closely related to any of the three stone dimensions.

Materials and Methods: A retrospective study of all patients who had a computed tomography scan with renal colic protocol at our hospital between January 1, 2016 and June 30, 2017. The maximum axial diameter, axial width, maximum coronal length and location of all stones were recorded. Patients were followed up for at least six months to ensure that the stones had been expelled or surgically removed.

Results: Ninety patients spontaneously passed their ureteric calculus, and 80 patients received surgery in this study. If the patients who received surgery within three days of diagnosis were excluded, the spontaneous stone passage rate was $81.1 \%$. Of the 90 patients with spontaneous stone passage, 38.9\%, 15.6\% and 6.7\% patients had stones with at least a 1.0, 1.5 and $2.0 \mathrm{~mm}$ difference between the maximum axial and coronal dimensions, respectively. Within the subset of calculi that passed within 90 days, these elongated calculi required between 3 and 6 less median days to pass than the more rounded calculi, although this was not statistically significant.

Conclusion: This study showed that in isolation, the individual dimension of a ureteric calculus did not significantly affect the time required to pass the calculus spontaneously. Elongated-shaped calculi were not common in this study.

Keywords: Tomography, spiral computed, renal colic, ureterolithiasis, urinary calculi

\section{Introduction}

The ureteric stone passage rate with medical expulsive therapy is related to stones' size. However, radiological reporting of ureteric stone sizes is not standardised. It is not uncommon to see some reports based on either axial or coronal dimensions, or both. Published studies commonly reported the maximum diameter in any plane as the official stone size. However, some studies used the maximum axial diameter as the official stone size (1-
3). Coll et al. (3) also proved that the maximum stone diameter in the axial plane, not just the maximum diameter in any plane, correlated with the chance of spontaneous stone passage. The presumed reasoning behind this is that the axial surface area is more relevant than the coronal/sagittal length as it passes through the cylinder-shaped ureter. This factor becomes more of a dilemma in cases of an oval-shaped/elongated stone with individual dimensions bordering the size limit that determines primary surgical intervention or medical expulsive therapy.

Correspondence: Dwayne Chang MD, Rockingham General Hospital, Clinic of Urology, Rockingham, Western Australia Phone: (+618) 95994000 E-mail: Dwayne.Chang@health.wa.gov.au ORCID-ID: orcid.org/0000-0003-4268-2479 Received: 07.04.20 Accepted: 03.07.20

Cite this article as: Chang D, Lozinskiy M, Jacques A, Kuan M. The Effect of Individual Stone Dimensions on Stone Passage Rates. J Urol Surg 2021;8(1):18-22.

${ }^{\circ}$ Copyright 2020 by the Association of Urological Surgery / Journal of Urological Surgery published by Galenos Publishing House. 
No study was found in the current literature to show a relationship between the chance of spontaneous stone passage and all three individual stone dimensions in the same study or the same cohort of patients. Hence, this study's primary objective was to determine the relationship between the three ureteric stone dimensions and their passage rates. Our secondary objective was to identify ureteric stones with an oval/ elongated shape that were expelled and determine whether the stone passage rate was preferentially related to any of the three stone dimensions.

\section{Materials and Methods}

We performed a retrospective study of all patients who had a computed tomography (CT) scan with a renal colic protocol at our hospital between January 1, 2016, to June 30, 2017, that diagnosed ureterolithiasis. No ethics committee approval was sought for this study because of its retrospective and observational (non-interventional) nature. Patients with renal calculus only (i.e. without ureteric calculus) were excluded. All CT examinations were performed with a Philips Brilliance 64 CT scanner (Koninklijke Philips N.V., Amsterdam, the Netherlands). Axial images were obtained from the top of the kidneys to the base of the bladder using a 3 and 1-1.5 mm thickness for thick and thin slices, respectively. A pitch of 0.891 or 1.173 was used for thick slices depending on the patient's body habitus as per our Radiology Department's protocol. Coronal and sagittal views were obtained with 3 and $5 \mathrm{~mm}$-thick slices, respectively. These digital radiological images were displayed using the AGFA IMPAX version 6.5.3.1509 PACS (picture archiving and communication system) software (Agfa-Gevaert N.V., Mortsel, Belgium). Images and reports of all scans were reviewed to ascertain whether ureteric calculi were found. Axial measurements of each stone were taken from the thin axial slices, not the thick slices. The maximum axial diameter, axial width and maximum coronal length of each imaged stone was also measured to the nearest $0.5 \mathrm{~mm}$ under magnification for this study by an author (DC), irrespective of the reported measurements on the official radiology report. This is because most of the official radiology reports at our institution did not contain all three dimensions. Hospital identification number, date of birth, gender, date of initial and follow-up scan and all stones' dimensions and location were recorded. Follow-up scans or subsequent surgeries for each patient were recorded for up to six months after initial diagnosis to determine whether the calculus has been expelled or removed, and the number of days between scans. If there was no record of follow-up scans or surgery, each of these patients was contacted by phone to check if there was a follow-up scan or surgery done outside of the public hospital system, whether they have seen the calculus expelled and the estimated date this occurred. If they reported the stone passage in the urine (either by catching it or viewing it), they did not require a repeat imaging study. If they have not had any follow-up scan and have not seen the calculus expelled, they were advised to have a urinary tract imaging study to exclude a retained calculus regardless of whether they had any renal colic. Patients with incomplete records of followup imaging or surgical intervention were excluded from this study. The principles of the Declaration of Helsinki (2013) were followed when conducting this study.

\section{Statistical Analysis}

Descriptive summaries were based on frequencies and means for categorical and continuous data, respectively. Time to event data was summarised using means and medians with corresponding 95\% confidence intervals. Days to pass stones were estimated using Kaplan-Meier survival probabilities, with log-rank tests to test statistical differences between survival curves for individual dimensions. Datasets were analysed using Stata 15.0 (StataCorp LLC, College Station, Texas) and IBM SPSS version 24.0 (Armonk, NY). P-values $<0.05$ were considered statistically significant.

\section{Results}

During the study period, 170 patients satisfied the inclusion and exclusion criteria of this study. In total, 90 patients had spontaneously passed their ureteric stones during this study, which included 61 men and 29 women with a mean age of 51.6 years. Among them, 76 patients passed their calculi within 90 days. One of them had bilateral vesicoureteric junction (VUJ) ureteric calculi. Eighty patients required surgical treatment for their ureteric stones, eight of which were bilateral stones or two stones in one ureter. The rate of spontaneous stone passage was $52.9 \%$ (90/170). Of these 80 operated patients, 59 were operated within three days of diagnosis. Retrospectively, this indicated that 111 patients were given at least four days for spontaneous stone passage, which was successful in 90 (81.1\%) and failed in $21(18.9 \%)$ patients who eventually required surgical calculi removal. The majority (44/91, with separate bilateral VUJ stones) passed stones that were located at the VUJ (48.4\%) and the distal ureter (26.4\%) at initial diagnosis. The median number of days required between the initial and follow-up scan or the date when the patient reportedly expelled the calculus was 38.0 days (range, 1-312 days). If only stones that passed within 90 days were analysed, the median number of days for stone passage was 32 days. The median size of expelled calculi in the maximum axial diameter, axial width and maximum coronal length were $4.1,3.0$ and $4.0 \mathrm{~mm}$, respectively. On the other hand, all operated calculi's median size dimensions were 6.8, 5.0 and $7.0 \mathrm{~mm}$, respectively.

In all patients who passed their calculi, there was no statistically significant difference in the time required to pass each calculus (Figure 1). Calculi with maximum axial, axial width and maximum 
coronal diameters up to the median size required a similar number of days to pass spontaneously, with no statistically significant difference. This finding was also present for calculi with dimensions greater than the median size. A similar result was found for the subset of patients who passed their calculi within 90 days (Figure 2).

The stones of interest in this study were those with a more elongated or oval-shaped profile. These stones were identified by selecting stones with at least a 1.0, 1.5 and $2.0 \mathrm{~mm}$ difference between the maximum axial and coronal dimensions. Respectively, there were 35 (38.9\%), 14 (15.6\%) and 6 (6.7\%) patients with such stones that passed spontaneously in the study. The log-rank test did not reveal a statistical correlation between the difference in maximum axial and coronal dimensions and the time required to pass these stones (Tables 1 and 2). Within the subset of calculi that passed within 90 days, these elongated calculi required between three and six less median days to pass than the more rounded calculi. However, this finding was not statistically significant across the calculi subset with a 1.0, 1.5 and $2.0 \mathrm{~mm}$ difference between the maximum axial and maximum coronal diameters (Tables 1 and 2).
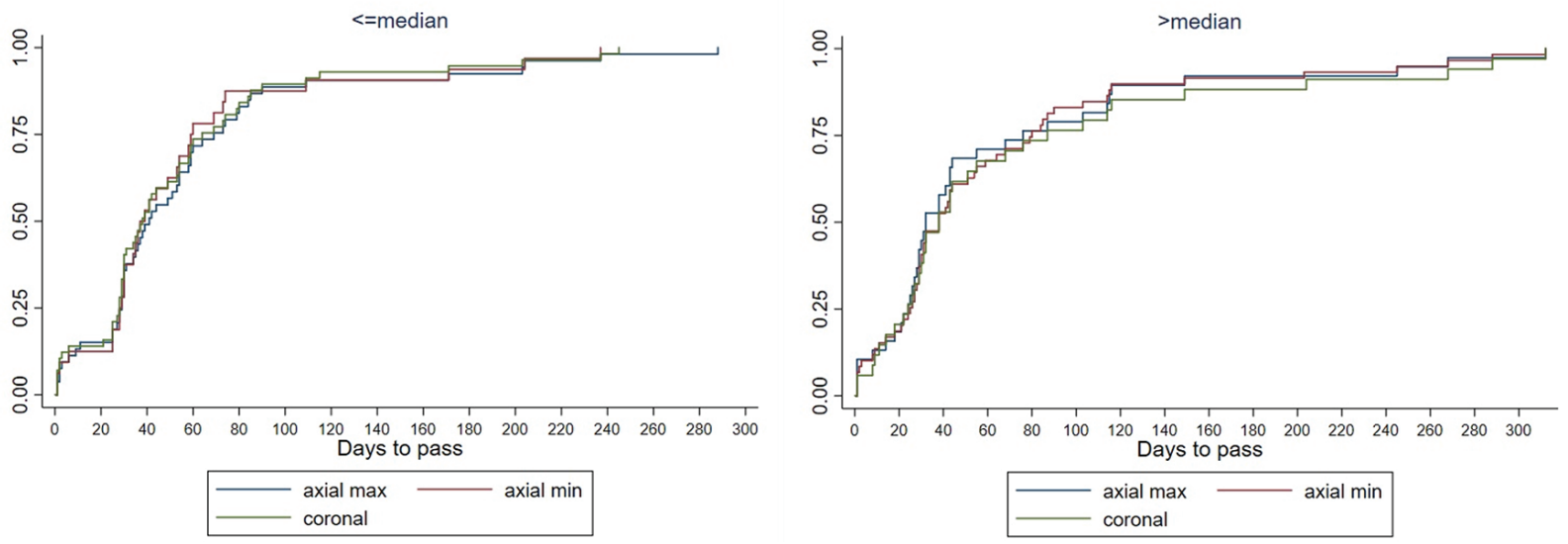

Figure 1. Kaplan-Meier inverted survival curve showing days to pass calculus $(n=91)$ by dimension for calculi up to and exceeding median cut-off sizes
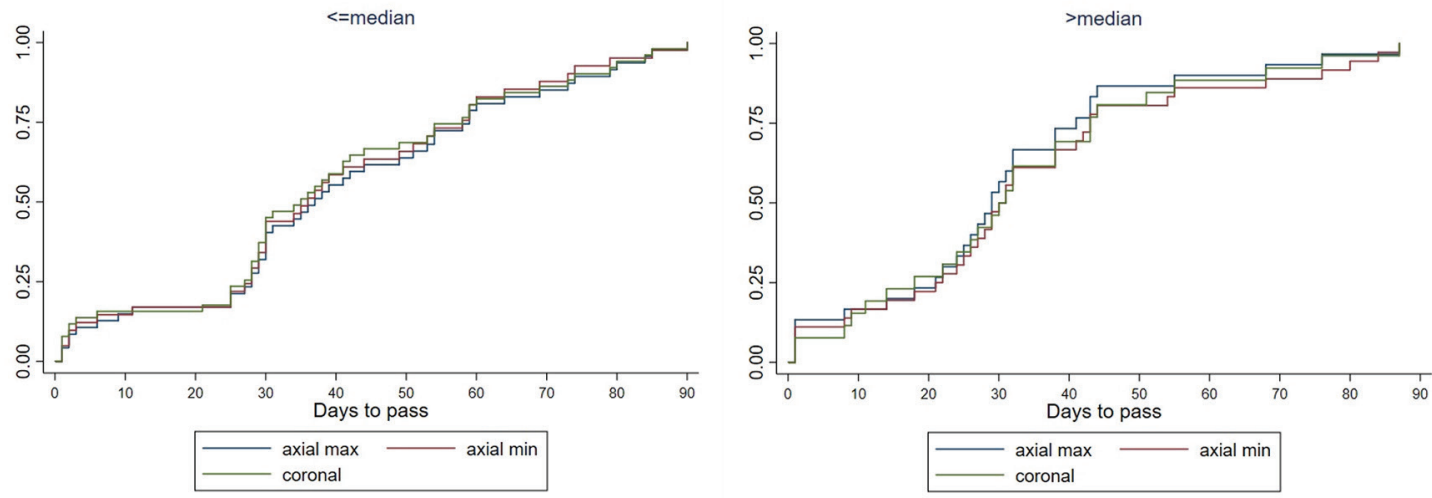

Figure 2: Kaplan-Meier inverted survival curve showing days to pass a stone (within 90 days, $n=77$ ) by dimension for calculi up to and exceeding median cutoff sizes (log-rank test: $p=0.966)$ 


\begin{tabular}{|c|c|c|c|c|}
\hline Classification & $\begin{array}{l}\text { Difference in stone } \\
\text { dimension* }\end{array}$ & $\begin{array}{l}\text { Number of stones (Total } \\
n=91)^{* *}\end{array}$ & $\begin{array}{l}\text { Median }(95 \% \mathrm{Cl}) \text { number of days to } \\
\text { pass all stones }\end{array}$ & p-value \\
\hline Elongated & $\geq 1 \mathrm{~mm}$ & 35 & $31(19.4-42.6)$ & 0.869 \\
\hline Not elongated & $<1.5 \mathrm{~mm}$ & 76 & $37(29.5-44.5)$ & 0.232 \\
\hline Elongated & $\geq 2 \mathrm{~mm}$ & 6 & $29(0.0-85.4)$ & 0.575 \\
\hline
\end{tabular}

Table 2. Log-rank testing of time taken to pass stones of elongated and non-elongated shapes for stones that passed within 90 days of diagnosis

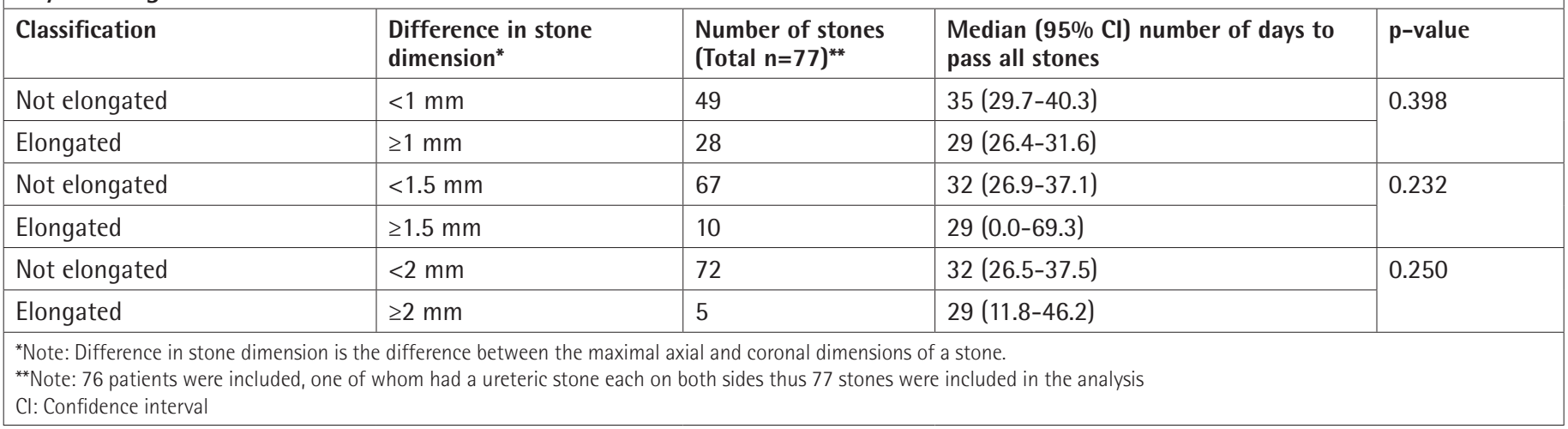

Our study attempted to determine which of the three dimensions of oval-shaped/elongated stones was the best predictive factor of stone passage time. Although a weak statistical correlation was found between each of the three dimensions of ureteric stones and the time required to expel them, we need to consider that a larger sample may be required to show any statistical significance considering the relatively small number of stones of particular interest in this study (stones with at least a 1.0-2.0 $\mathrm{mm}$ difference between maximum axial and coronal diameters). This can also explain the weak correlation between the individual dimensions of any passed stone and the time required for stone passage as logically there should be a strong correlation between these variables as shown in previous studies $(3,9)$. Radiological reporting of stone size needs to be standardised towards the stone dimension with the strongest correlation to the chance of spontaneous passage, be it the maximum diameter in any or a specific plane. Although this study has attempted to identify which of these dimensions is the most important, the answer has not been scientifically proven.

\section{Study Limitations}

One of the limitations of this retrospective study was potential recall bias from reporting of the date that patients expelled their stone spontaneously. Another important note was that on CT, the axial views generally had thinner slices than the sagittal and coronal views; thus, the degree of precision in measurements may differ. We also assumed that urgent surgery would have occurred within three days of the diagnosis to differentiate patients allocated to spontaneous passage instead of urgent surgery from the outset. With a prospective design, one can more accurately record the exact number of patients allocated to passage or surgery from the outset and standardise the medical expulsive therapy regimen. Future studies may improve recall accuracy and data collection by prospectively employing more stringent and frequent follow-ups. This surveillance may address issues we encountered, such as uncompliant patients (ie, those who forgot about their stones, did not check their urine and missed follow-up scans and appointments). A prospective study design with weekly follow-up phone calls is one means of improving compliance and data collection. Conventionally, follow-up scans were requested to be done four weeks after the initial diagnosis. However, we found that patient non-compliance, availability of imaging services and non-standardised management by frontline doctors might have been a factor for the irregular periods between the initial and follow-up scans. An audit with our methodology is another tool to pick-up patients who are lost to follow-up. Standardisation of management protocols by frontline doctors 
in future prospective-design studies might reduce the risk of this confounding variable.

\section{Conclusion}

This study showed that in isolation, any individual dimension of a ureteric calculus did not have a statistically significant effect on the time required to pass the calculus spontaneously over other calculus dimensions. Calculi of interest (elongated-shaped) were not common in this study. Although there was an inclination that these calculi pass earlier than the more rounded calculi, future prospective studies with a larger cohort might provide a more definitive answer. Although this study has not proved a preferential relationship for a particular stone dimension, this question remains unanswered because of the lack of studies specifically studying this topic in the current medical literature. If future studies prove an association, radiological reporting should include this particular stone dimension as a standard. This information is clinically relevant as it may improve the decision-making process for stones with borderline sizes and ensure that patients receive evidence-based treatment.

\section{Ethics}

Ethics Committee Approval: No ethics committee approval was sought for this study because of its retrospective and observational (non-interventional) nature.

Informed Consent: Retrospective study.

Peer-review: Externally and internally peer-reviewed.

\section{Authorship Contributions}

Concept: D.C., M.L., M.K., Design: D.C., M.L., M.K., Data Collection or Processing: D.C., Analysis or Interpretation: D.C., M.L., A.J., M.K., Literature Search: D.C., M.L., A.J., M.K., Writing: D.C., M.L., A.J., M.K.
Conflict of Interest: No conflict of interest was declared by the authors.

Financial Disclosure: The authors declared that this study received no financial support.

\section{References}

1. Yap WW, Belfield JC, Bhatnagar P, Kennish S, Wah TM. Evaluation of the sensitivity of scout radiographs on unenhanced helical CT in identifying ureteric calculi: a large UK tertiary referral centre experience. Br J Radiol 2012;85:800-806.

2. Mokhless I, Zahran AR, Youssif M, Fouda K, Fahmy A. Factors that predict the spontaneous passage of ureteric stones in children. Arab J Urol 2012:10:402-407.

3. Coll DM, Varanelli MJ, Smith RC. Relationship of spontaneous passage of ureteral calculi to stone size and location as revealed by unenhanced helical CT. AJR Am J Roentgenol 2002;178:101-103.

4. Nazim SM, Ather MH, Khan N. Measurement of ureteric stone diameter in different planes on multidetector computed tomography - impact on the clinical decision making. Urology 2014;83:288-292.

5. Furyk JS, Chu K, Banks C, Greenslade J, Keijzers G, Thom O, Torpie T, Dux C, Narula R. Distal Ureteric Stones and Tamsulosin: A Double-Blind, PlaceboControlled, Randomized, Multicenter Trial. Ann Emerg Med 2016;67:86-95. e2.

6. Pickard R, Starr K, MacLennan G, Lam T, Thomas R, Burr J, McPherson G, McDonald A, Anson K, N'Dow J, Burgess N, Clark T, Kilonzo M, Gillies K, Shearer K, Boachie C, Cameron S, Norrie J, McClinton S. Medical expulsive therapy in adults with ureteric colic: a multicentre, randomised, placebocontrolled trial. Lancet 2015;386:341-349.

7. Foell $\mathrm{K}$, Ordon M, Ghiculete $\mathrm{D}$, Lee JY, Honey RJ, Pace KT. Does baseline radiography of the kidneys, ureters, and bladder help facilitate stone management in patients presenting to the emergency department with renal colic? J Endourol 2013;27:1425-1430.

8. Kishore $T A$, Pedro RN, Hinck $B$, Monga M. Estimation of size of distal ureteral stones: noncontrast CT scan versus actual size. Urology 2008;72:761-764.

9. Tchey DU, Ha YS, Kim WT, Yun SJ, Lee SC, Kim WJ. Expectant Management of Ureter Stones: Outcome and Clinical Factors of Spontaneous Passage in a Single Institution's Experience. Korean J Urol 2011;52:847-851. 